\title{
Bounds for singular values of a block companion matrix
}

\author{
Manuela S. Silva and Teresa P. de Lima
}




\title{
BOUNDS FOR SINGULAR VALUES OF A BLOCK COMPANION MATRIX
}

\author{
MANUELA S. SILVA AND TERESA P. DE LIMA
}

Received 17 March, 2005

\begin{abstract}
Upper bounds for the squares of the singular values of a block companion matrix are derived. The principal tools applied are matricial and matrix norms and inequalities concerning the spectral radius of nonnegative matrices.
\end{abstract}

1991 Mathematics Subject Classification: 15A18, 15A42

Keywords: singular values, matricial norms, matrix norms, block companion matrix

\section{INTRODUCTION}

Singular value decomposition (SVD) was established for real matrices in 1870 by E. Beltrami and C. Jordan, and for complex squares matrices by Autonne. Later, in 1939, Eckart and Yong computed the SVD of any rectangular matrices. The study of singular values has been receiving a lot of attention since they constitute one of the most useful tools in handling, reliably, various problems which arise in a wide variety of application areas, such as linear algebra [3], statistical analysis, signal and image processing [4], electrical network theory [4], control and systems theory [3] and biomedical engineering [2]. On the other hand, we must remember that scalar companion matrices appear frequently in dynamic system theory: their special structure allows converting higher-order scalar difference and differential equations to state space form as well as simplifies theoretical considerations as for example feedback analysis.

Besides, note that any completely observable single-output system can be transformed into a companion form system [1]. Hence, the motivation for this work lies in the fact that our research is mainly concerned with system theory and we verify that these two concepts (singular values and companion matrices) assume an important role in the area referred to. The paper [9], where the authors, making use of the spectral radius of a matrix, matricial and matrix norms, derived bounds for the singular values of a block companion matrix, attracted our attention. Here, taking into account that the singular values different from one, of the right block companion 
matrix

associated to the matrix polynomial

$$
C_{R}=\left(\begin{array}{cccc}
0 & \ldots & 0 & -A_{0} \\
I & \ldots & 0 & -A_{1} \\
\ldots \ldots & \ldots & \ldots . . \\
0 & \ldots & I & -A_{m-1}
\end{array}\right)
$$

$$
M(\lambda)=A_{m} \lambda^{m}+A_{m-1} \lambda^{m-1}+\cdots+A_{2} \lambda^{2}+A_{1} \lambda+A_{0}
$$

are the positive square roots of the latent roots of the $\lambda$-matrix

$$
Q(\lambda)=I \lambda^{2}-(\mathcal{A}+I) \lambda+A_{0}^{T} A_{0},
$$

we simplify the upper bound for the squares of the singular values of the $C_{R}$ presented in [9].

\section{Preliminaries}

For the materialization of our purpose we need to remember the following definitions and results.

Definition 1 ([8]). Let $A_{0}, A_{1}, \ldots, A_{m-1}$ and $X \in Q_{n}(\mathbb{R})$. The polynomial

$$
M(X)=A_{m} X^{m}+A_{m-1} X^{m-1}+\cdots+A_{2} X^{2}+A_{1} X+A_{0}
$$

is called a matrix polynomial of degree $m$.

Definition 2 ([8]). If in (2.1) $X$ is a scalar matrix, i. e., $X=\lambda I(\lambda \in \mathbb{C})$, then

$$
M(X)=A_{m} \lambda^{m}+A_{m-1} \lambda^{m-1}+\cdots+A_{2} \lambda^{2}+A_{1} \lambda+A_{0}
$$

is designated by lambda-matrix.

Definition 3 ([8]). Given a matrix polynomial

$$
M(X)=X^{m}+A_{m-1} X^{m-1}+\cdots+A_{2} X^{2}+A_{1} X+A_{0},
$$

the right block companion matrix associated with $M(X)$ is a matrix $C_{R} \in M_{m}\left(Q_{n}\right)$ defined by

$$
C_{R}=\left(\begin{array}{cccc}
0 & \ldots & 0 & -A_{0} \\
I & \ldots & 0 & -A_{1} \\
\ldots \ldots & \ldots & \ldots \\
0 & \ldots & I & -A_{m-1}
\end{array}\right)
$$

Theorem 1 ([3]). Let $A \in M_{m}\left(Q_{n}\right)$ of rank $r$. Then there exist orthogonal matrices $U \in Q_{m}(\mathbb{R})$ and $V \in Q_{n}(\mathbb{R})$ such that

$$
U^{T} A V^{T}=\left(\begin{array}{cc}
\Sigma_{1} & 0 \\
0 & 0
\end{array}\right)=\Sigma,
$$

where $\Sigma_{1}=\operatorname{diag}\left(\sigma_{1}, \sigma_{2}, \ldots, \sigma_{r}\right)$ and $\sigma_{i}>0, i=1,2, \ldots, r$. The factorization

$$
A=U \Sigma V^{T}
$$


is called the singular value decomposition of the matrix $A$ and the principal elements of $\Sigma_{1}$ are the singular values of $A$.

Remark 1. Recall that $\sigma_{1}^{2}, \sigma_{2}^{2}, \ldots, \sigma_{r}^{2}$ are the eigenvalues different from zero of $A^{T} A$ and $A A^{T}$.

Definition 4 ([8]). The latent roots of the lambda-matrix

$$
M(\lambda)=A_{m} \lambda^{m}+A_{m-1} \lambda^{m-1}+\cdots+A_{2} \lambda^{2}+A_{1} \lambda+A_{0}
$$

are the zeros of $\operatorname{det} M(\lambda)=0$.

Definition 5 ([10]). Let $A=\left(a_{i j}\right) \in Q_{n}(\mathbb{C})$. The maximum column sum matrix norm of $A$ is given by the formula

$$
\|A\|_{1}:=\max _{j} \sum_{i}\left|a_{i j}\right| .
$$

Finally, we need to bear in mind the following result which plays an important role in order to attain our aim:

Lemma 1 ([5]). The singular values of the block companion matrix $C_{R}$ are the square roots of the eigenvalues of the quadratic $\lambda$-matrix

$$
I \lambda^{2}-(\mathcal{A}+I) \lambda+A_{0}^{T} A_{0}, \quad \mathcal{A}=\sum_{j=0}^{m-1} A_{j}^{T} A_{j} .
$$

\section{RESULTS}

In this section, we are going to establish upper bounds for the squares of the singular values of the right block companion matrix $C_{R}$.

Proposition 1. The singular values $\sigma_{j}^{2}, j=1, \ldots, 2 n$, different from one, of the right block companion matrix $C_{R} \in M_{m}\left(Q_{n}\right)$ associated with the matrix polynomial

$$
M(\lambda)=A_{m} \lambda^{m}+A_{m-1} \lambda^{m-1}+\cdots+A_{2} \lambda^{2}+A_{1} \lambda+A_{0},
$$

satisfy the following inequality:

$$
\sigma_{j}^{2} \leq\left(1+\max \left\{\|\mathcal{A}+I\|_{1}^{2},\left\|\left(A_{0}^{T} A_{0}\right)^{2}\right\|_{1}+\left\|(\mathcal{A}+I)^{2}\right\|_{1}\right\}\right)^{\frac{1}{2}} .
$$

Proof. First, we consider the block companion matrix $C_{R} \in M_{2}\left(Q_{n}\right)$ defined by

$$
C=\left(\begin{array}{cc}
0 & -A_{0}^{T} A_{0} \\
I & \mathcal{A}+I
\end{array}\right)
$$

associated with the matrix polynomial

$$
Q(\lambda)=I \lambda^{2}-(\mathcal{A}+I) \lambda+A_{0}^{T} A_{0},
$$


where

$$
\mathcal{A}=\sum_{j=0}^{m-1} A_{j}^{T} A_{j} .
$$

Consequently, since $(\mathcal{A}+I)^{T}=\mathcal{A}+I$, we get

$$
C^{T} C=\left(\begin{array}{cc}
I & \mathcal{A}+I \\
\mathcal{A}+I & \left(A_{0}^{T} A_{0}\right)^{2}+(\mathcal{A}+I)^{2}
\end{array}\right) .
$$

Further, we form the matrix $D=\operatorname{diag}\left[\|\mathcal{A}+I\|_{1} I, I\right]$ and take the norm $\|\cdot\|_{1}$ of each block of the matrix

$$
D^{-1} C^{T} C D=\left(\begin{array}{cc}
I & \|\mathcal{A}+I\|_{1}^{-1}(\mathcal{A}+I) \\
\|\mathcal{A}+I\|_{1}(\mathcal{A}+I) & \left(A_{0}^{T} A_{0}\right)^{2}+(\mathcal{A}+I)^{2}
\end{array}\right),
$$

obtaining the matricial norm of $D^{-1} C^{T} C D$ denoted by $\Phi_{1}\left(D^{-1} C^{T} C D\right)$.

We have

$$
\Phi_{1}\left(D^{-1} C^{T} C D\right)=\left(\begin{array}{cc}
1 & 1 \\
\|\mathcal{A}+I\|_{1}^{2} & \left\|\left(A_{0}^{T} A_{0}\right)^{2}+(\mathcal{A}+I)^{2}\right\|_{1}
\end{array}\right) .
$$

Taking into account the following relations concerning the spectral radius $\rho$ of a matrix, matricial and matrix norms $[9,11]$

$$
\begin{aligned}
& \rho(A) \leq\|A\|_{i}, \quad i=1, \infty, \\
& \rho(A) \leq\left(\rho\left(A^{T} A\right)\right)^{\frac{1}{2}} \leq\left(\rho\left(\Phi_{i}\left(A^{T} A\right)\right)\right)^{\frac{1}{2}} \leq\left\|\Phi_{i}\left(A^{T} A\right)\right\|_{j}^{\frac{1}{2}}, \quad i, j=1, \infty,
\end{aligned}
$$

we can write

$$
\begin{aligned}
& \rho\left(C^{T} C\right)=\rho\left(D^{-1} C^{T} C D\right) \\
& \leq \sqrt{\rho\left(\begin{array}{c}
1 \\
\|\mathscr{A}+I\|_{1}^{2}
\end{array} \quad\left\|\left(A_{0}^{T} A_{0}\right)^{2}+(\mathcal{A}+I)^{2}\right\|_{1}\right)} .
\end{aligned}
$$

Thus

$$
|\lambda| \leq\left\|\left(\begin{array}{cc}
1 & 1 \\
\|\mathcal{A}+I\|_{1}^{2} & \left\|\left(A_{0}^{T} A_{0}\right)^{2}+(\mathcal{A}+I)^{2}\right\|_{1}
\end{array}\right)\right\|_{1}^{\frac{1}{2}},
$$

or equivalently,

$$
|\lambda| \leq\left[1+\max \left\{\|\mathcal{A}+I\|_{1}^{2},\left\|\left(A_{0}^{T} A_{0}\right)^{2}\right\|_{1}+\left\|(\mathcal{A}+I)^{2}\right\|_{1}\right\}\right]^{\frac{1}{2}},
$$

where $\lambda$ denotes a latent root of $Q(\lambda)$. 
Remark 2. Note that the matrix on the right member of (3.4) is a square matrix of order two. So, evaluating directly the eigenvalues, we obtain

$$
|\lambda| \leq\left(\frac{p+\sqrt{p^{2}-4 \times(q-r)}}{2}\right)^{\frac{1}{2}},
$$

where $r=\|\mathcal{A}+I\|_{1}^{2}, p=1+\left\|\left(A_{0}^{T} A_{0}\right)^{2}+(\mathcal{A}+I)^{2}\right\|_{1}$, and

$$
q=\left\|\left(A_{0}^{T} A_{0}\right)^{2}+(\mathcal{A}+I)^{2}\right\|_{1},
$$

which provides a sharper bound but is not so practical for computing.

Finding bounds for the eigenvalues of a matrix polynomial is a problem to which many researchers have given contributions. In [5] a variety of lower and upper bounds were derived from block companion matrix, singular values, numerical radius, norms of the coefficient matrices of $M(\lambda)$, characteristic polynomial, fractional powers of norms and maximization over unit circle. We are going to apply some of these results to the quadratic $\lambda$-matrix

$$
Q(\lambda)=I \lambda^{2}-(\mathcal{A}+I) \lambda+A_{0}^{T} A_{0},
$$

where

$$
\mathcal{A}=\sum_{j=0}^{m-1} A_{j}^{T} A_{j},
$$

and to compare them with the estimate obtained in Proposition 1. Let us consider $Q_{U}(\lambda)=Q(\lambda)$ and

$$
Q_{L}(\lambda)=I \lambda^{2}-\left(A_{0}^{T} A_{0}\right)^{-1}(\mathcal{A}+I) \lambda+\left(A_{0}^{T} A_{0}\right)^{-1},
$$

the two $\lambda$-matrices associated with $Q(\lambda)$, whose block companion matrices are defined by the formulas

$$
C_{U}=\left(\begin{array}{cc}
0 & I \\
-A_{0}^{T} A_{0} & \mathcal{A}+I
\end{array}\right)
$$

and

$$
C_{L}=\left(\begin{array}{cc}
0 & I \\
-\left(A_{0}^{T} A_{0}\right)^{-1} & \left(A_{0}^{T} A_{0}\right)^{-1}(\mathcal{A}+I)
\end{array}\right),
$$

respectively. Using the norms of $C_{U}$ and $C_{L}$ [5], we obtain the estimates

$$
\begin{aligned}
\max \left\{\left\|\left(A_{0}^{T} A_{0}\right)^{-1}\right\|_{1}, 1+\|\left(A_{0}^{T} A_{0}\right)^{-1}\right. & \left.(\mathcal{A}+I) \|_{1}\right\}^{-1} \leq \sigma_{j}^{2} \\
& \leq \max \left\{1+\|\mathcal{A}+I\|_{1},\left\|A_{0}^{T} A_{0}\right\|_{1}\right\},
\end{aligned}
$$




$$
\begin{aligned}
\max \left\{1,\left\|\left(A_{0}^{T} A_{0}\right)^{-1}-\left(A_{0}^{T} A_{0}\right)^{-1}(\mathcal{A}+I)\right\|_{\infty}\right\}^{-1} & \leq \sigma_{j}^{2} \\
& \leq \max \left\{1,\left\|\left(A_{0}^{T} A_{0}\right)-(\mathcal{A}+I)\right\|_{\infty}\right\}
\end{aligned}
$$

and

$$
\begin{aligned}
\| I+\left(A_{0}^{T} A_{0}\right)^{-2}+\left(A_{0}^{T} A_{0}\right)^{-1}+(\mathcal{A}+I)^{2} & \left(A_{0}^{T} A_{0}\right)^{-1} \|_{2}^{-\frac{1}{2}} \leq \sigma_{j}^{2} \\
& \leq\left\|I+\left(A_{0}^{T} A_{0}\right)^{-2}+(\mathcal{A}+I)^{2}\right\|_{2}^{\frac{1}{2}} .
\end{aligned}
$$

The numerical radius of a matrix provides another upper bound for the eigenvalues of $Q(\lambda)$ as it has been shown in [5]. Invoking Lemma 2.11 we have

$$
\sigma_{j}^{2} \leq \frac{1}{2}\left(1+\|\mathcal{A}+I\|_{2}+\left\|\left(A_{0}^{T} A_{0}\right)-(\mathcal{A}+I)\right\|_{2}\right)
$$

In the same article, N. J. Higham and F. Tisseur by using norms of the coefficient matrices of $M(\lambda)$ obtained a generalization of the Cauchy bound. In our case, we get

$$
\sigma_{j}^{2} \leq \frac{1}{2}\left(\|\mathcal{A}+I\|+\left(\|\mathcal{A}+I\|^{2}+4\left\|A_{0}^{T} A_{0}\right\|\right)^{\frac{1}{2}}\right)
$$

and

$$
\sigma_{j}^{2} \geq \frac{1}{2}\left(-\|\mathcal{A}+I\|+\left(\|\mathcal{A}+I\|^{2}+4\left\|\left(A_{0}^{T} A_{0}\right)^{-1}\right\|^{-1}\right)^{\frac{1}{2}}\right) .
$$

It should be mentioned that the same upper bound, based in matrix inequalities, spectral radius and spectral norm can be found in [7]. Other methods involving fractional powers of norms and maximization over unit circle yield bounds for the eigenvalues of $M(\lambda)$. For example, the application of Lemma 4.1 from [5] leads one to the following bounds for the squares of the singular values of $C_{R}$ :

$$
\begin{aligned}
\left(1+\left\|\left(A_{0}^{T} A_{0}\right)^{-1}\right\|\right)^{-1} \min \left\{\|\mathcal{A}+I\|^{-1}, 1\right\} & \leq \sigma_{j}^{2} \\
& \leq 2 \max \left\{\|\mathcal{A}+I\|,\left\|A_{0}^{T} A_{0}\right\|^{\frac{1}{2}}\right\}
\end{aligned}
$$

Any of these upper bounds require the computation of matrix norms subordinate to vector norms. From the similarities presented by the above majorization relations, we think that there is not an upper bound that outperforms all the others. The accuracy of a bound depends of the range of the elements of the coefficient matrices of $M(\lambda)$. Assume that the maximum in (3.1), (3.5) and (3.6) is attained by the first term and $\|\mathcal{A}+I\|_{1}$ is of a large order of magnitude. Thus, the first bound provides the same estimate for $\sigma^{2}$ as the one obtained by (3.5) and by the Cauchy bound. However, it 
is sharpper than the easily expressed bound given by (3.6). The following example makes it clear what we have referred to.

Example 1. Let us consider the $5 \times 5$ matrix polynomial

$$
M(\lambda)=A_{m} \lambda^{m}+A_{m-1} \lambda^{m-1}+\cdots+A_{2} \lambda^{2}+A_{1} \lambda+A_{0}
$$

of degree $m=9$ whose coefficient matrices are of the form

$$
A_{i}=10^{i-2} \text { randn, } \quad i=0,1, \ldots, 8 ; \quad \text { and } A_{9}=I,
$$

where "randn" denotes a random matrix with elements removed from a Normal distribution $N(0,1)$. In our experiment, performed by using Matlab 5.3, the maximal value of the eigenvalues of the $\lambda$-matrix

$$
Q(\lambda)=I \lambda^{2}-(\mathcal{A}+I) \lambda+A_{0}^{T} A_{0}
$$

is $1.3909 \times 10^{13}$. The upper bounds presented in (3.1), (3.5) as well as the Cauchy bound yield an estimate of $1.5458 \times 10^{13}$. On the other hand, using (3.6), we verify that $\sigma_{j}^{2} \leq 3.09168 \times 10^{13}$.

Example 2. Observe that some robustness measures for state space models in control and system theory such as nearness to uncontrollability and nearness to instability require the computation of singular values [6]. More concretely, singular values provide bounds on the nearness to instability of closed loop systems of the form

$$
x(k+1)=(A+B F) x(k),
$$

where

$$
A=\left(\begin{array}{cc}
0 & I_{n-1} \\
a_{1} & A
\end{array}\right)
$$

with $A=\left(\begin{array}{lll}a_{2} & \ldots & a_{n}\end{array}\right), B=\left(\begin{array}{c}0_{n-1} \\ 1\end{array}\right)$, and $F$ the feedback matrix. In this case, considering the set of all stable matrices

$$
P_{S}=\left\{A \in \mathbb{R}^{n \times n}: \operatorname{Re}(\lambda)<0 \text { for all eigenvalues of } A\right\},
$$

Kenny and Laub [6] pointed out that the distance from $A+B F$ to the complement of $P_{S}, d\left(A+B F, P_{S}^{C}\right)$, provides a means of measuring nearness to instability and that

$$
d\left(A+B F, P_{S}^{C}\right)=\min _{\operatorname{Re} \lambda \geq 0} \sigma_{\min }(A+B F-\lambda I) \leq \sigma_{\min }(A+B F),
$$

where $\sigma_{\min }(\cdot)$ is the smallest singular value of a matrix. So, information about the location of singular values is useful. Let us consider the discrete-time linear invariant system

$$
x(k+1)=A x(k)+B u(k),
$$


where

$$
A=\left(\begin{array}{cc|cc|cc}
0 & 0 & 1 & 0 & 0 & 0 \\
0 & 0 & 0 & 1 & 0 & 0 \\
\hline 0 & 0 & 0 & 0 & 1 & 0 \\
0 & 0 & 0 & 0 & 0 & 1 \\
\hline-1 & 0 & -1 & 0 & 1 & -1 \\
1 & 1 & 0 & 1 & 0 & -2
\end{array}\right), \quad B=\left(\begin{array}{c}
0 \\
0 \\
\hline 0 \\
0 \\
\hline 0 \\
1
\end{array}\right),
$$

and $F=\left(\begin{array}{ll|ll|ll}-2 & 0 & 0 & 0 & 0 & 0\end{array}\right)$. So,

$$
A+B F=\left(\begin{array}{cc|cc|cc}
0 & 0 & 1 & 0 & 0 & 0 \\
0 & 0 & 0 & 1 & 0 & 0 \\
\hline 0 & 0 & 0 & 0 & 1 & 0 \\
0 & 0 & 0 & 0 & 0 & 1 \\
\hline-1 & 0 & -1 & 0 & 1 & -1 \\
-1 & 1 & 0 & 1 & 0 & -2
\end{array}\right)
$$

In order to apply the result obtained, we need to find the matrices $\left(A_{0}^{T} A_{0}\right)^{2}$ and $\mathcal{A}+$ $I$. We verify that $\left(A_{0}^{T} A_{0}\right)^{2}=\left(\begin{array}{cc}5 & -3 \\ -3 & 2\end{array}\right)$, and $\mathcal{A}+I=\left(\begin{array}{cc}5 & -2 \\ -2 & 8\end{array}\right), \mathcal{A}=\sum_{j=0}^{2} A_{j}^{T} A_{j}$, which allows us to obtain $\|\mathcal{A}+I\|_{1}^{2}=100,\left\|\left(A_{0}^{T} A_{0}\right)^{2}\right\|_{1}=8$, and $\left\|(\mathcal{A}+I)^{2}\right\|_{1}=$ 94. In this way, by Proposition 1 , we conclude that

$$
\sigma_{j}^{2} \leq \sqrt{103}, \quad j=1,2,3,4 .
$$

Observe that the maximal square singular value, $\sigma_{\max }^{2}(A+B F)=9.5809$.

\section{CONCLUDING REMARKS}

In this paper, from the relationship between singular values of a block companion matrix and the latent roots of a lambda matrix and using inequalities (3.2) and (3.3), we obtain upper bounds for the squares of the singular values of the right block companion matrix $C_{R}$. We would like to emphasize that the simplicity of inequality (3.1) is a consequence of the application of Lemma 1 that enables us to solve our problem beginning with a quadratic lambda-matrix, instead of one of order $m$. Finally, we hope that this work could be useful in the study of the controllability and stability of systems in scalar companion form.

\section{Acknowledgement}

The authors express their gratitude to the referee's suggestions which helped to improve their initial version of the paper. 


\section{REFERENCES}

[1] S. Barnett, Introduction to Mathematical Control Theory, ser. Oxford Applied Mathematics and Computing Science Series. Oxford: Clarendon Press, 1975.

[2] D. Callaerts, B. De Moor, J. Vandewalle, W. Sansen, G. Vantrappen, and J. Janssens, "Comparison of SVD methods to extraction of the foetal electrocardiogram from cutaneous electrode signals," Med. Biol. Eng. Comp., vol. 28, pp. 217-224, 1990.

[3] B. N. Datta, Numerical Linear Algebra and Applications. Pacific Grove, CA: Brooks/Cole Publishing Co., 1995.

[4] B. De Moor, J. Staar, and J. Vandewalle, "Oriented energy and oriented signal-to-signal ratio concepts in the analysis of vector sequences and time series," in SVD and Signal Processing, Algorithms and Architectures. Elsevier Science, 1988, pp. 209-232.

[5] N. J. Higham and F. Tisseur, "Bounds for eigenvalues of matrix polynomials," Linear Algebra Appl., vol. 358, pp. 5-22, 2003, special issue on accurate solution of eigenvalue problems (Hagen, 2000).

[6] C. Kenney and A. J. Laub, "Controllability and stability radii for companion form systems," Math. Control Signals Systems, vol. 1, no. 3, pp. 239-256, 1988.

[7] F. Kittaneh, "Bounds for the zeros of polynomials from matrix inequalities," Arch. Math. (Basel), vol. 81, no. 5, pp. 601-608, 2003.

[8] P. Lancaster, Lambda-Matrices and Vibrating Systems, ser. International Series of Monographs in Pure and Applied Mathematics. Oxford: Pergamon Press, 1966.

[9] T. Lima and J. Vitória, "Bounds for the singular values of block companion matrices," Linear Algebra Appl., vol. 170, pp. 225-228, 1992.

[10] M. Parodi, La localisation des valeurs caractéristiques des matrices et ses applications. Préface de H. Villat, ser. Traité de Physique Théorique et de Physique Mathématique, XII. Paris: GauthierVillars, 1959.

[11] F. Robert, "Étude et utilisation des normes vectorielles en analyse numérique linéaire," Thèse, Grenoble, 1968.

Authors' addresses

Manuela S. Silva

Universidade de Coimbra, Faculdade de Economia, Av. Dias da Silva, 165, 3000 Coimbra, Portugal

E-mail address: nelinha@fe.uc.pt

\section{Teresa P. de Lima}

Universidade de Coimbra, Faculdade de Economia, Av. Dias da Silva, 165, 3000 Coimbra, Portugal

E-mail address: tpl@fe.uc.pt 